\title{
Critical Droplets and Metastability for a Glauber Dynamics at Very Low Temperatures
}

\author{
E. Jordão Neves and Roberto H. Schonmann $\star$ \\ Instituto de Matemática e Estatística, Universidade de São Paulo, Caixa Postal 20570, 01498. \\ São Paulo, SP, Brasil
}

Received November 25, 1989

\begin{abstract}
We consider the metastable behavior in the so-called pathwise approach of a ferromagnetic spin system with a Glauber dynamics in a finite two dimensional torus under a positive magnetic field in the limit as the temperature goes to zero. First we consider the evolution starting from a single rectangular droplet of spins +1 in a sea of spins -1 . We show that small droplets are likely to disappear while large droplets are likely to grow; the threshold between the two cases being sharply defined and depending only on the external field. This result is used to prove that starting from the configuration with all spins down $(-1)$ the pattern of evolution leading to the more stable configuration with all spins up $(+\underline{1})$ approaches, as the temperature vanishes, a metastable behavior: the system stays close to -1 for an unpredictable time until a critical square droplet of a precise size is eventually formed and nucleates the decay to $+\underline{1}$ in a relatively short time. The asymptotic magnitude of the total decay time is shown to be related to the height of an energy barrier, as expected from heuristic and mean field studies of metastability.
\end{abstract}

\section{Introduction}

The problem of metastability has continued to attract a great deal of attention during recent years (see for instance the update $[\mathrm{PL}]$ ). Here we consider the two dimensional nearest neighbor ferromagnetic Ising Model, with an external magnetic field, evolving according to a Glauber dynamics (i.e., a reversible spin flip dynamics). In the limit as the temperature goes to zero, for fixed finite volume, we show that this model presents the essential features that one associates with metastability. The main result is roughly as follows. If the external field is small and positive, the system, when started from the configuration with all spins down, behaves as

* Partially supported by CNPq. Part of this work was done while RHS was visiting Rome, supported by an agreement between $\mathrm{CNPq}$ and $\mathrm{CNR}$ 
if it where in a steady state for a very long time until a critical droplet of a very precise shape and size is formed and nucleates, in a relatively short time, the evolution to the configuration with all spins up. The system then stays close to this configuration for a much longer time. The size of the critical droplet and the typical asymptotic value for the time of the decay agree with the predictions made on heuristic grounds (one can see the phenomenon as an escape from a potential well). The decay time is sharply defined in the proper scale for each realization of the process since the time needed for the final passage from all spins down to all spins up is much shorter than the decay time. But since this decay is nucleated by the unlikely appearance of a critical droplet, the rescaled decay time varies from realization to realization of the process and has in fact a distribution which becomes exponential in the limit as the temperature vanishes, characterizing its unpredictability. The apparent stability around the configuration with all spins down is evinced by the fact that in the scale of the decay time the process converges in distribution to a very simple pure jump process which stays in the configuration with all spins down and then suddenly jumps to the one with all spins up where it remains forever.

The characterization of metastable behavior in the fashion above was introduced in the paper [CGOV] to which we refer the reader for more through discussion on the motivation of this picture as capturing the basic aspects of metastability. This so-called "pathwise approach" was further developed and applied in several situations in the papers: [KN], [Sch], [NCK], [GOV], [COP], [MOS], [EGJL] and [Bra].

No one has succeeded so far in establishing some sort of metastability in this pathwise sense for Glauber dynamics at fixed finite low temperature in the thermodynamic limit. In fact for doing it one would certainly have to let the external magnetic field go to zero as the volume grows, since otherwise nucleation would occur throughout the system, which would behave in a very deterministic and smooth way. A first step in this direction would be the analysis of this system with no external magnetic field. One may then expect, for instance, the average value of the spin (the empirical magnetization) to converge (in the proper sense) as the volume grows and after rescaling the time to a symmetric Markovian pure jump process with only two states, $-m^{*}$ and $+m^{*}=$ the spontaneous magnetization.

Here we study a Glauber dynamics in a different regime. We investigate the behavior of the system in finite volume and at very low temperature, so that the correlation length is much larger than the system. Clearly our results still hold if we let the volume grow slowly enough as the temperature decreases to zero. But since we did not try to estimate how fast the volume can grow, we postpone this question to a future investigation. From the physical point of view, the important point is to establish for realistic volumes (of the order of Avogadro's number) how low the temperature must be for our asymptotic results to be good approximations of the actual behavior of the system.

\section{Results}

We consider the two dimensional nearest neighbor ferromagnetic Ising Model on a finite torus (periodic boundary conditions): $\Lambda_{N}=\{1,2, \ldots, N\}^{2}$. The Hamiltonian 
is written as

$$
H_{N}(\sigma)=-\frac{1}{2} \sum_{\langle x, y\rangle} \sigma(x) \sigma(y)-\frac{h}{2} \sum_{x} \sigma(x),
$$

where $\sigma(x) \in\{-1,+1\}$, the first sum runs over the pairs of nearest neighbors sites of $\Lambda_{N}$, counting each pair only once, and the second is taken over $\Lambda_{N}$. We will always consider $h>0$.

At inverse temperature $\beta \geqq 0$ the Gibbs measure $\mu_{N}$ is given by

$$
\begin{aligned}
\mu_{N}(\sigma) & =\left(Z_{N}\right)^{-1} \exp \left(-\beta H_{N}(\sigma)\right), \\
Z_{N} & =\sum_{\sigma} \exp \left(-\beta H_{N}(\sigma)\right) .
\end{aligned}
$$

For $N$ and $h>0$ both fixed and $\beta \rightarrow \infty, \mu_{N}$ concentrates its mass on the configuration with all spins up, which we will denote by $+\underline{1}$. We will also write -1 for the configuration with all spins down.

By a Glauber dynamics we mean a spin flip dynamics which is reversible with respect to the Gibbs measure (This is called a stochastic Ising model in [Lig].). We will only consider the Glauber dynamics where the spin at site $x$, when the configuration is $\eta \in\{-1,+1\}^{\Lambda_{N}}$, flips at rate

$$
c(x, \eta)=\left\{\begin{array}{ll}
1 & \text { if } \Delta_{x} H(\eta) \leqq 0 \\
\exp \left(-\beta\left(\Delta_{x} H(\eta)\right)\right) & \text { otherwise }
\end{array},\right.
$$

where $\Delta_{x} H(\eta)=H\left(\eta^{x}\right)-H(\eta)$, with

$$
\eta^{x}(y)=\left\{\begin{array}{lll}
\eta(y) & \text { if } & x \neq y \\
-\eta(y) & \text { if } & x=y
\end{array}\right.
$$

For each initial configuration $\eta$ these rates define a continuous time Markov process $\left(\sigma_{t}^{n}, t \geqq 0\right)$, such that, at $t=0, \sigma_{t}^{\eta}=\eta$ with probability one and, for $\xi \neq \zeta$ and $\varepsilon>0$,

$$
P\left(\sigma_{t+\varepsilon}^{n}=\xi \mid \sigma_{t}^{\eta}=\zeta\right)=\left\{\begin{array}{ll}
c(x, \zeta) \varepsilon+o(\varepsilon) & \text { if } \xi=\zeta^{x} \\
o(\varepsilon) & \text { otherwise }
\end{array} \text { for some } x \in \Lambda_{N}\right.
$$

One can construct this process by choosing at rate $N^{2}$ a random site in $\Lambda_{N}$ and then flipping the spin $\sigma(x)$ with probability $c(x, \sigma)$, where $\sigma$ is the current configuration. The imbedded Markov chain obtained is precisely the Metropolis algorithm for simulating the Gibbs measure.

Now we define, for each $\eta \in\{-1,+1\}^{\Lambda_{N}}$ and $A \subset\{-1,+1\}^{\Lambda_{N}}$, the hitting time

$$
T^{\eta}(A)=\inf \left\{t \geqq 0: \sigma_{t}^{\eta} \in A\right\} .
$$

If $A=\{\xi\}$ we write simply $T^{\eta}(\xi)$. If $\eta$ is omitted it is -1 and if $A$ is omitted it is $\{+\underline{1}\}$.

Our main goal is to describe the behavior of the system when it starts from -1 until it reaches $+\underline{1}$ for $N$ and $h$ both fixed and $\beta$ going to infinity. As we will see the answer depends on the values of $h$ and $N$.

If $h>4$ any spin -1 will flip at rate 1 even if its four neighbors are also -1 . On the other hand any spin +1 will flip with a vanishing rate as $\beta \rightarrow \infty$. Let $\left(\bar{\sigma}_{t}^{\eta}, t \geqq 0\right)$ be the process starting from $\eta$ in which each spin -1 flips independently of the others with rate 1 and each spin +1 does not flip at all. It is easy to prove that: 
Proposition 1. If $h>4$ then for any $\eta \in\{-1,+1\}^{\Lambda_{N}},\left(\sigma_{t}^{\eta}, t \geqq 0\right)$ converges as $\beta \rightarrow \infty$ to $\left(\bar{\sigma}_{t}^{\eta}, t \geqq 0\right)$ in the sense that we can construct these two processes on the same probability space in such a way that, for any $t_{0}<\infty$,

$$
\lim _{\beta \rightarrow \infty} P\left(\sigma_{t}^{\eta} \neq \bar{\sigma}_{t}^{n} \text {, for some } t \in\left[0, t_{0}\right]\right)=0 .
$$

In particular $T$ converges in distribution to the maximum of $N^{2}$ independent unit mean exponential random variables and $\lim _{\beta \rightarrow \infty} E T=\sum_{k=1}^{N^{2}} \frac{1}{k}$. So there is no
metastability in the case $h>4$.

When $h<4$ the first spin flip starting from the configuration -1 increases the energy by $4-h$ so that the total rate is $N^{2} \exp (-\beta(4-h))$ and as a consequence $T$ diverges to infinity as $\beta$ increases.

If $2<h<4$ and $N \geqq 3$ after reaching the state with only one spin +1 three things can happen. With rate 1 the system goes back to $-\underline{1}$, with rate 4 it goes to a configuration with two neighbors +1 spins and with rate $\left(N^{2}-5\right) \exp (-\beta(4-h))$ it goes to other configuration with two +1 spins. As the third possibility has vanishing rate (as $\beta \rightarrow \infty$ ) only the first two are important. If the system goes back to $-\underline{1}$ everything starts anew but if it gets to a configuration with two neighbors +1 spins then both of them are flipping back with vanishing rates $(\exp (-\beta(-2+h)))$ while its neighbors are flipping from -1 to +1 with rates 1 . So the pair of neighbors spin +1 forms our first example of a critical droplet that nucleates the passage from $-\underline{1}$ to $+\underline{1}$.

This behavior illustrates the general picture of the passage from $-\underline{1}$ to $+\underline{1}$ for small magnetic field $h$. The process spends most of the time in -1 , making many quick trips into other configurations and going back, until it reaches a critical configuration from where it goes to $+\underline{1}$ in a short time (we are using the scale given by $E T$ to decide if a time is short or long).

When $0<h<2$ a droplet with only two neighbors +1 surrounded by -1 spins is no longer critical as it tends to shrink and disappear. We will find nevertheless that the concept of critical droplets is well defined and that small droplets tend to shrink while big ones tend to grow. The separation between the two behaviors is sharp and the critical droplet turns out to be a square of size close to $2 / h$.

Theorem 1 below characterizes the critical droplet as it describes what happens when the starting configuration is a rectangular droplet of spins +1 depending on "how big it is."

Let $\mathscr{R}$ be the set of configurations with all spins -1 except for those in a rectangle $l_{1} \times l_{2}$ which are +1 , with $l_{1}$ and $l_{2}$ less than $N-1$. For $\eta \in \mathscr{R}$ define $l=l(\eta)=\min \left(l_{1}, l_{2}\right)$. Before stating Theorem 1 we need two definitions.

Given $\varepsilon>0$ and starting from a configuration $\eta \in \mathscr{R}$ we say that the event $S_{\varepsilon}(\eta)$, called "the droplet shrinks $\varepsilon$-regularly," happens if " $T^{\eta}(-1)<T$, from time 0 up to time $T^{\eta}(-1)$ all spins which where -1 at time 0 are still -1 and all spins +1 form a single cluster; also $\exp (\beta(h(l-1)-\varepsilon))<T^{\eta}(-1)<\exp (\beta(h(l-1)+\varepsilon))$." The notion of cluster is the usual one from percolation [Kes]: the clusters of spin +1 are the maximal sets of sites which can be connected by chains of neighboring sites of spins +1 .

Given $\varepsilon>0$ and starting from a configuration $\eta \in \mathscr{R}$ we say that the event $G_{\varepsilon}(\eta)$, called "the droplet grows $\varepsilon$-regularly," happens if " $T^{\eta}<T^{\eta}(-1)$, from time 0 up to time $T^{\eta}$ the number of spins +1 is at least $l_{1} l_{2}-l+1$ and they form a single 
cluster such that the minimal rectangle that contains all of them also contains the original one defined by $\eta$; also $\exp (\beta(2-h-\varepsilon))<T^{\eta}<\exp (\beta(2-h+\varepsilon))$."

Theorem 1. Suppose that $0 \leqq h<2$ and $\eta \in \mathscr{R}$.

a) If $l(\eta)<2 / h$ and $\varepsilon>0$ then

$$
\lim _{\beta \rightarrow \infty} P\left(S_{\varepsilon}(\eta)\right)=1
$$

b) If $l(\eta)>2 / h$ and $\varepsilon>0$ then

$$
\lim _{\beta \rightarrow \infty} P\left(G_{\varepsilon}(\eta)\right)=1 .
$$

The value $2 / h$ for the threshold corresponds to what one expects heuristically when one computes the energy of a configuration $\eta \in \mathscr{R}$ in which the +1 spins form a square of side $l$ :

$$
H(\eta)-H(-\underline{1})=4 l-l^{2} h=: e(h, l),
$$

as for fixed $h$ its maximum is attained at $l=2 / h$. We define $L=\lceil 2 / h\rceil=$ smallest integer larger than $2 / h$. If $2 / h$ is not an integer, then $L$ is the size of the critical droplet. The case when $2 / h$ is an integer is somewhat particular as, in this case, a $\eta \in \mathscr{R}$ with $l(\eta)=2 / h$ can either grow or shrink with non-vanishing probabilities as $\beta$ increases. This does not cause any real difficulty but to facilitate the exposition we will not consider this case.

If $N$ is smaller or barely larger than $L$, the passage from $-\underline{1}$ to $+\underline{1}$ could be nucleated by the appearance of a ring of spins +1 around the torus $\Lambda_{N}$ and not by the appearance of a critical square droplet $L \times L$. To concentrate on the more interesting case, we will suppose from now on that $N$ is large enough. We did not try to verify how small $N$ can be for the nucleation still to occur by the appearance of the critical square droplet, instead we consider the following technical condition:

Standard Case: $0<h<2,2 / h$ is not an integer and $N>N(h):=L^{2}+1$.

The next theorem characterizes the metastable behavior of $\left(\sigma_{t}^{-1}, t \geqq 0\right)$ in a pathwise sense.

Theorem 2. In the standard case

a)

$$
\frac{T}{E T} \rightarrow \tau \quad \text { in distribution as } \beta \rightarrow \infty
$$

where $\tau$ is a unit mean exponential random variable.

b) The rescaled process $\left(\sigma_{s E T}^{-1}, s \geqq 0\right)$ converges as $\beta \rightarrow \infty$ to a Markovian pure jump process $\left(\xi_{s}, s>0\right)$ which stays in -1 for a unit mean exponential time and then jumps to +1, where it stays forever. This convergence is in the sense of finite dimensional distributions, i.e., for every integer $k \geqq 0$ and $0 \leqq t_{1} \leqq t_{2} \leqq \cdots \leqq t_{k}$ the distribution of $\left(\sigma_{t_{i} E T}^{-\frac{1}{E}}, i=1, \ldots, k\right)$ converges to that of $\left(\xi_{t_{i} E T}^{-\frac{1}{E}}, i=1, \ldots, k\right)$.

Even if $N$ is big enough it is possible, in principle, that the critical droplet is never seen in the passage from $-\underline{1}$ to $+\underline{1}$. This would happen, for instance, if the process escapes from $-\underline{1}$ by the formation of two subcritical droplets that coalesce into a supercritical one. The next theorem shows that this is not likely in the 
standard case and that in fact the decay from the metastable state is, with probability going to one as $\beta$ increases, through the appearance of a critical droplet. The next theorem also gives a sharp estimate on the magnitude of $T$. Before stating it we need some definitions.

Let $\mathscr{G}$ be the set of configurations in $\mathscr{R}$ in which the +1 spins form a square droplet of size $L$. Let $\mathscr{P}$ be the set of configurations with all spins -1 except for a single cluster of spins +1 formed by a rectangle $L \times(L-1)$ together with a spin +1 attached to one of its larger sides. The droplets in configurations of $\mathscr{P}$ can be considered as proto-critical droplets since they can either grow to a critical one or shrink to a rectangle $L \times(L-1)$, that is subcritical, both with non-vanishing probabilities as $\beta \rightarrow \infty$ and this decision takes place in times of order one.

For each $\eta \in \mathscr{P}$ we have

$$
H(\eta)-H(-\underline{1})=4 L-\left(L^{2}-L+1\right) h=e(h, L)+(L+1) h=: \Gamma(h) .
$$

Remark. Direct computations show that $\Gamma(h)$ is a continuous and strictly decreasing function of $0<h<2$. As $h \rightarrow 0, \Gamma(h) \sim 4 / h$.

Theorem 3. In the standard case
a) $\lim _{\beta \rightarrow \infty} \frac{1}{\beta} \log T=\Gamma(h)$ in probability,
b) $\lim _{\beta \rightarrow \infty} \frac{1}{\beta} \log E T=\Gamma(h)$,
c) $\lim _{\beta \rightarrow \infty} P(T(\mathscr{G})<T)=1$,
d) $\lim _{\beta \rightarrow \infty} P(T(\mathscr{P})<T)=1$.

This theorem can also be extended to the case $2<h<4$ and $N$ large if one defines $\mathscr{G}$ as the set of configurations with all spins down, except for two neighbor sites, where the spins are up, and $\mathscr{P}$ as the set of configurations with a single spin up. Then $\Gamma(h)=4-h$.

After proving Theorem 1 in the next section, we will see that it has as a consequence the possibility of dividing the set of all configurations into three non-empty sets $\mathscr{A}, \mathscr{B}$ and $\mathscr{C}$ such that $\mathscr{A}$ and $\mathscr{C}$ are, respectively, the basins of attraction of $-\underline{1}$ and $+\underline{1}$, while starting from $\mathscr{B}$ the system can go to $\mathscr{A}$, or $\mathscr{C}$, both with non-vanishing probabilities as $\beta$ increases. More precisely we have.

Proposition 2. In the standard case the set of configurations can be partitioned into three non-empty sets $\mathscr{A}, \mathscr{B}$ and $\mathscr{C}$ such that

a) If $\eta \in \mathscr{A}$ then

$$
\lim _{\beta \rightarrow \infty} P\left(T^{\eta}(-\underline{1})<T^{\eta}, T^{\eta}(-\underline{1})<\exp (\beta(2-h))\right)=1 .
$$

b) If $\eta \in \mathscr{C}$ and $\varepsilon>0$ then

$$
\lim _{\beta \rightarrow \infty} P\left(T^{\eta}<T^{\eta}(-\underline{1}), T^{\eta}<\exp (\beta(2-h+\varepsilon))\right)=1 .
$$

c) If $\eta \in \mathscr{B}$ then

$$
\begin{aligned}
& \liminf _{\beta \rightarrow \infty} P\left(T^{\eta}(-\underline{1})<T^{\eta}\right)>0, \\
& \liminf _{\beta \rightarrow \infty} P\left(T^{\eta}<T^{\eta}(-\underline{1})\right)>0,
\end{aligned}
$$


and for every $\varepsilon>0$

$$
\lim _{\beta \rightarrow \infty} P\left(T^{\eta}(\{-1,+1\})<\exp (\beta(2-h+\varepsilon))\right)=1 .
$$

Precise definitions will be given in Sect. 3, but roughly speaking, these sets are clearly as follows: configurations in $\mathscr{A}$ contain only small droplets of spins up, configurations in $\mathscr{C}$ contain at least one large droplet and in $\mathscr{B}$ we have droplets of such shapes and sizes that they may either grow or shrink (for instance, the configurations in $\mathscr{P})$.

One can easily verify that $\Gamma(h)>2-h$, when $0<h<2$. Hence we have that, in the standard case, whatever the starting configuration, the system quickly relaxes to $-\underline{1}$ or $+\underline{1}$ in the scale of time given by ET.

\section{Characterization of the Critical Droplet}

In this section we prove Theorem 1 , describe the sets $\mathscr{A}, \mathscr{B}$ and $\mathscr{C}$ that partition the set of configurations and prove Proposition 2.

First we identify the mechanism that is responsible for the existence of critical droplets. It seems to us that even at the heuristic level this mechanism has escaped people's attention.

In order to avoid particularities suppose $h<1$ and that the process starts at a configuration $\eta \in \mathscr{R}$ such that $l(\eta)>3$. In $\eta$ all the spins are flipping at vanishing rates as $\beta$ increases and the largest of them, $\exp (-\beta h)$, are those of the spins at the four corners of the rectangle of +1 spins. So, very likely, the first change corresponds to a flip of one of these spins. But then the new -1 spin flips back at rate one and the rectangle is recovered with great probability. It could happen, however, with probability of order $\exp (-\beta h)$ that a second corner disappears before the replacement of the first one. Extending this reasoning one sees then that disregarding flips of spins +1 which are surrounded by more than two spins +1 , the number of spins -1 in the region occupied originally by the spins +1 behaves in a way which resembles a birth and death process, births occurring with rate of order $\exp (-\beta h)$ and deaths with rate of order 1. Until a time of the order $\exp (-\beta h k)$ the droplet will have up to $k$ spins, but not more, missing at some moments. Hence if only this "corner erosion mechanism" were at work, one would have the disappearance of a whole line on the droplet border in a time of order $\exp (\beta h(l-1))$, since the last +1 spin in a line at the border has positive probability of flipping in time of order one. Opposing to this tendency there are -1 spins adjacent to the original rectangle and neighbor to the droplet flipping at rate $\exp (\beta(-2+h))$. The result of the competition between these two tendencies decides what happens to this droplet. The comparison between $\exp (\beta h(l-1))$ and $\exp (\beta(2-h))$ originates the threshold $l=2 / h$.

If $l<2 / h$ the erosion dominates and before the appearance of a +1 outside the original rectangle one has the disappearance of a whole line in such a way that a smaller configuration in $\mathscr{R}$ is reached. Since now the erosion is easier this behavior repeats itself until -1 is reached.

If $l>2 / h$ then before a time much smaller than $\exp (\beta h(l-1))$ all the sides of the original rectangle have at least one +1 spin since there is not enough time to complete the erosion. But in a time of order $\exp (\beta(2-h))$, much smaller than 
$\exp (\beta(h(l-1)))$ for $\beta$ large, one of the -1 spins adjacent to a +1 spin at the border of the droplet will flip, originating a protuberance. One has now at least one -1 spin neighbor both to the protuberance and to the droplet flipping at rate 1 . So before time $\exp (\beta(2-h+\varepsilon))$ for $\varepsilon>0$ one has a protuberance with two +1 spins together and, with great probability, an extra slice will be completed reaching a bigger rectangular configuration. As the tendency to grow is larger for bigger droplets, the same arguments holds again until $+\underline{1}$ is reached.

Before going into the proof based on this heuristics we observe that if $0<h<1$ even when $l>2 / h$ it is clear that the derivative at time 0 of the expected number of +1 spins is negative for large $\beta$ as the corner erosion mechanism dominates for short times. In [HMM] a non-rigorous study of critical droplets for a different Glauber dynamics (for the same Hamiltonian) was carried on, with the analysis being based on the sign of the derivative at time zero of the number of spins in the droplet. Our analysis here can be adapted to the dynamics considered in that paper, yielding again the conclusion that this derivative at time zero is in fact not a good indicator for the behavior of the droplet for long times.

In the proof of Theorem 1 we will need the following result:

Lemma 1. Let $\left\{X_{t}\right\}_{t \geqq 0}$ be $a$ birth and death process on $\{0,1, \ldots\}$ with birth rate $\delta<1$ and death rate 1 . If $X_{0}=0$, define

$$
\Theta_{\delta}=\inf \left\{t \geqq 0: X_{t}=k\right\} .
$$

Then, for every $k \geqq 1$ and $\varepsilon>0$, we have

$$
\lim _{\delta \rightarrow 0} P\left(\delta^{-k+\varepsilon}<\Theta_{\delta}<\delta^{-k-\varepsilon}\right)=1 .
$$

Proof. The unique invariant measure of this process is $\rho(n)=(1-\delta) \delta^{n}, n=0,1,2, \ldots$. Let $\left\{X_{t}^{\rho}\right\}_{t \geqq 0}$ be the same process with initial position chosen with distribution $\rho$. We couple the processes starting from different states in such a way that both evolve independently until they meet and then evolve together. Then

$$
\begin{aligned}
P\left(\Theta_{\delta} \leqq\right. & \left.\delta^{-k+\varepsilon}\right) \leqq \\
& +P\left(X_{0}^{0} \neq X_{0}^{\rho} \geqq k \text { for some } t \in\left\{0, \delta^{\varepsilon / 2}, 2 \delta^{\varepsilon / 2}, \ldots,\left\lceil\delta^{-k+\varepsilon / 2}\right\rceil \delta^{\varepsilon / 2}\right\}\right) \\
& +P\left(X_{t}^{\rho}<k \text { for every } t \in\left\{0, \delta^{\varepsilon / 2}, 2 \delta^{\varepsilon / 2}, \ldots,\left\lceil\delta^{-k+\varepsilon / 2}\right\rceil \delta^{\varepsilon / 2}\right\}, \Theta_{\delta} \leqq \delta^{-k+\varepsilon}\right) \\
\leqq & (\delta)+\left(\delta^{k}\left\lceil\delta^{-k+\varepsilon / 2}\right\rceil\right)+\left(1-e^{-\delta^{\varepsilon / 2}}\right)
\end{aligned}
$$

that goes to zero as $\delta \rightarrow 0$. (For estimating the second probability we used stationarity and for the third one the fact that $\Theta_{\delta}$ is a stopping time and the rates of jump of our birth and death process are bounded above by 1.)

Observe now that

$$
P\left(\Theta_{\delta}<1\right) \geqq e^{-1}\left(1-e^{-\delta / k}\right)^{k},
$$

since this is a lower bound for the probability that from time 0 to $1\left(X_{t}^{0}\right)$ makes $k$ jumps up and no jump down. Since it is easier to reach $\{k, k+1, k+2, \ldots\}$ if the starting position is not 0 (for a proof one can use a coupling as above), it follows that

$$
P\left(\Theta_{\delta} \geqq \delta^{-k-\varepsilon}\right) \leqq\left(1-e^{-1}\left(1-e^{-\delta / k}\right)^{k}\right)^{\delta-k-\varepsilon},
$$

which goes to zero as $\delta \rightarrow 0$.

We now proceed to the proof of Theorem 1. 
Define two auxiliary processes $\left(\hat{\sigma}_{t}^{\eta}, t \geqq 0\right)$ and $\left(\check{\sigma}_{t}^{\eta}, t \geqq 0\right)$ with $P\left(\hat{\sigma}_{0}^{\eta}=\eta\right)=P\left(\check{\sigma}_{0}^{\eta}=\eta\right)=1$ for $\eta \in\{-1,+1\}^{\Lambda_{N}}$ and respective rates $\hat{c}(x, \eta)$ and $\check{c}(x, \eta)$, given by

$$
\hat{c}(x, \eta)= \begin{cases}0 & \text { if } \eta(x)=\eta(y) \text { for at least three } \\ c(x, \eta) & \text { sites } y \text { which are neighbors of } x \\ \text { otherwise. }\end{cases}
$$

and

$$
\check{c}(x, \eta)= \begin{cases}0 & \text { if } \eta(x)=\eta(y)=1 \text { for at least three } \\ c(x, \eta) & \text { sites } y \text { which are neighbors of } x,\end{cases}
$$

For $\eta \in \mathscr{R},\left(\hat{\sigma}_{t}^{\eta}\right)$ is the " corner mechanism." In this dynamics, the droplet can never grow, since protuberances cannot appear and it can only lose spins at the corners, which can also be recovered. But once a complete slice is lost, it is lost forever.

In $\left(\check{\sigma}_{t}^{\eta}\right)$ the droplet shrinks only by the "corner mechanism," but it can grow, since protuberances may appear.

In this and the coming sections we will make repeated use of monotonicity properties of $\left(\sigma_{t}^{\eta}\right),\left(\hat{\sigma}_{t}^{\eta}\right)$ and $\left(\check{\sigma}_{t}^{\eta}\right)$, related to attractiveness (see Sect. 2 of Chap. III of [Lig]). For this purpose we introduce the following partial order on $\{-1,+1\}^{\Lambda_{N}}$ :

$$
\eta \leqq \zeta \quad \text { iff } \eta(x) \leqq \zeta(x) \text { for every } x \in \Lambda_{N} .
$$

The following is true then by Theorem 1.5 of Chap. III of [Lig]. If $\eta \leqq \zeta$, then we can construct a coupling between the processes $\left(\sigma_{t}^{\eta}\right)$ and $\left(\sigma_{t}^{\zeta}\right)$ such that

$$
P\left(\sigma_{t}^{\eta} \leqq \sigma_{t}^{\zeta} \text { for all } t \geqq 0\right)=1 .
$$

Analogous statements hold for $\left(\hat{\sigma}_{t}\right)$ and $\left(\check{\sigma}_{t}\right)$.

We now couple $\left(\sigma_{t}^{\eta}\right),\left(\hat{\sigma}_{t}^{\eta}\right)$ and $\left(\check{\sigma}_{t}^{\eta}\right)$ with the so-called Basic or Vasershtein coupling (see Sect. 1 of Chap. III of [Lig] for a precise definition; informally speaking, this coupling makes the processes agree as much as possible). As another consequences of Theorem 1.5, Chap. III of [Lig] we have that for all $\xi$ and $\zeta$ such that $\xi \leqq \zeta$

$$
P\left(\hat{\sigma}_{t}^{\xi} \leqq \breve{\sigma}_{t}^{\zeta} \text { for all } t \geqq 0\right)=1 .
$$

Also these two new processes approximate the original one in the sense that for all $\eta \in \mathscr{R}$ and $\delta>0$

$$
\begin{aligned}
& \lim _{\beta \rightarrow \infty} P\left(\sigma_{t}^{\eta}=\hat{\sigma}_{t}^{\eta} \text { for all } t \in\left[0, e^{\beta(2-h-\delta)}\right]\right)=1, \\
& \lim _{\beta \rightarrow \infty} P\left(\sigma_{t}^{\eta}=\check{\sigma}_{t}^{\eta} \text { for all } t \in\left[0, e^{\beta(2+h-\delta)}\right]\right)=1
\end{aligned}
$$

as follows from the fact that the time until the first difference between $\left(\hat{\sigma}_{t}^{\eta}\right)$ (respectively $\left(\check{\sigma}_{t}^{\eta}\right)$ ) and $\left(\sigma_{t}^{\eta}\right)$ appears is dominated by an exponential time with mean $N^{-2} e^{\beta(2-h)}$ (respectively $N^{-2} e^{\beta(2+h)}$ ).

For $\eta \in\{-1,+1\}^{\Lambda_{N}}$ define $T_{+} \eta$ as the configuration obtained from $\eta$ by flipping all the spins -1 with at least two opposite neighbors. Define also $T_{-} \eta$ as the configuration obtained from $\eta$ by flipping all the spins +1 with at least three 
opposite neighbors. Applying iteratively $T_{+}$(respectively $T_{-}$) on $\eta$ we obtain an increasing (respectively decreasing) sequence of configurations that becomes constant after a finite number of applications. Denote by $\bar{\eta}$ (respectively $\underline{\eta}$ ) this final configuration.

If $0<h \leqq 2$, each one of the flips defining $T_{+}$and $T_{-}$does not increase the energy and therefore occurs with rate 1 for our Glauber dynamics and also for the two new dynamics. So one has:

Lemma 2. If $0<h \leqq 2$

$$
\begin{aligned}
& \inf _{\beta \geqq 0} \inf _{\eta \in\left\{-1,+1 \Lambda_{N}\right.} P(A)=: \alpha_{+}>0, \\
& \inf _{\beta \geqq 0} \inf _{\eta \in\{-1,+1\}^{\Lambda_{N}}} P(B)=: \alpha_{-}>0,
\end{aligned}
$$

where $A$ and $B$ are the following events:

$$
\begin{aligned}
& A:=\left\{\sigma_{s}^{\eta} \leqq \sigma_{t}^{\eta} \text { and } \check{\sigma}_{s}^{\eta} \leqq \check{\sigma}_{t}^{\eta} \text { for every } 0 \leqq s \leqq t \leqq 1 ; \sigma_{1}^{\eta}=\check{\sigma}_{1}^{\eta}=\bar{\eta}\right\}, \\
& B:=\left\{\sigma_{s}^{\eta} \geqq \sigma_{t}^{\eta} \text { and } \hat{\sigma}_{s}^{\eta} \geqq \hat{\sigma}_{t}^{\eta} \text { for every } 0 \leqq s \leqq t \leqq 1 ; \sigma_{1}^{\eta}=\hat{\sigma}_{1}^{\eta}=\underline{\eta}\right\} .
\end{aligned}
$$

If $\eta \in \mathscr{R}$ we can suppose without loss of generality that we have $l_{1}$ and $l_{2}$ with $l(\eta)=l_{1} \leqq l_{2}$ such that all the spins in $\eta$ are -1 except those inside the rectangle $R=\left\{1, \ldots l_{1}\right\} \times\left\{1, \ldots l_{2}\right\}$. We also define the slices of $\mathscr{R}$ to be the sets $H_{j}=\left\{1, \ldots l_{1}\right\} \times\{j\}$ and $V_{i}=\{i\} \times\left\{1, \ldots l_{2}\right\}$ with $j \in\left\{1, \ldots l_{2}\right\}$ and $i \in\left\{1, \ldots l_{1}\right\}$.

For the proof of part (a) of the theorem, take $\eta \in \mathscr{R}$ and define $\hat{\Theta}_{1}^{\eta}$ as the first time that the process $X_{t}:=\left|\left\{x \in H_{1}: \hat{\sigma}_{t}^{\eta}=-1\right\}\right|$ reaches $\{l-1\}$, where $l=l(\eta)$.

Lemma 3. For $\eta \in \mathscr{R}$ and $\delta>0$,

$$
\lim _{\beta \rightarrow \infty} P\left(\hat{\Theta}_{1}^{\eta}<\exp (\beta(h(l-1)+\delta))\right)=1 .
$$

Proof. Before $\hat{\Theta}_{1}^{\eta}$, the process $X_{t}$ increases by 1 with rate greater than $e^{-\beta h}$ and decreases by 1 with rate smaller than $N^{2}$. So a comparison between $X_{t}$ and a birth and death process with these rates together with Lemma 1 yields the result.

Suppose now that $l<2 / h$ and consider the times

$$
t_{i}=i \exp [\beta(h(l-1)+\varepsilon / 2)], \quad i=1,2, \ldots
$$

with $\varepsilon>0$ such that $h(l-1)+\varepsilon<2-h-\varepsilon$. Define $\Omega_{1}$ as the event that, for $\left(\hat{\sigma}_{t}^{\eta}\right)$ starting at configuration $\eta$, i) $\hat{\Theta}_{1}^{\eta}<t_{1} / 2$ and ii) after $\hat{\Theta}_{1}^{\eta}$ up to $t_{1}$ the last remaining spin +1 in $H_{1}$ flips before the flipping of any spin -1 .

For part (i) in the definition of $\Omega_{1}$ we use Lemma 3 and for (ii) we use Lemma 2 to conclude that there exists $\beta_{0}<\infty$ such that for $\beta>\beta_{0}$,

$$
P\left(\Omega_{1}\right) \geqq \alpha_{-} / 2 \text {. }
$$

Using the Markov property and monotonicity we have that for large $\beta$ the probability that the droplet will lose a slice in the process $\left(\hat{\sigma}_{t}^{\eta}\right)$ before time $\exp (\beta(2-h-\varepsilon))>\exp (\beta(h(l-1)+\varepsilon))$ is greater than

$$
1-\left(1-\left(\alpha_{-} / 2\right)\right)^{\exp (\beta \varepsilon / 2)}
$$

that goes to one as $\beta$ increases.

Using (6) and repeating the arguments above each time the droplet loses a slice, 
we see that

$$
\lim _{\beta \rightarrow \infty} P\left(T^{\eta}(-\underline{1})<T^{\eta}, \quad T^{\eta}(-\underline{1})<\exp (\beta(2-h-\varepsilon))\right)=1 .
$$

One can now easily complete the proof of part (a) of the theorem, using (6) again and estimates similar to those above for the process $\left(\hat{\sigma}_{t}^{\eta}\right)$. We leave this straightforward work to the reader.

For part (b) consider $\eta \in \mathscr{R}$ with $l=l(\eta)$ and define the hitting times $\breve{\Theta}^{\eta}$ and $\widehat{\Theta}_{2}^{\eta}$ of $\{l-1\}$ for the processes $Y_{t}:=\left|\left\{x \in R: \check{\sigma}_{t}^{\eta}(x)=-1\right\}\right|$ and $Z_{t}:=\left|\left\{x \in R: \hat{\sigma}_{t}^{\eta}(x)=-1\right\}\right|$, respectively.

Lemma 4. For $\eta \in \mathscr{R}$ and $\delta>0$,

$$
\lim _{\beta \rightarrow \infty} P\left(\check{\Theta}^{\eta}>\exp (\beta(h(l-1)-\delta))\right)=1 .
$$

Proof. By (5) we have that $Y_{t} \leqq Z_{t}$ and then

$$
P\left(\check{\Theta}^{\eta}>\exp (\beta(h(l-1)-\delta))\right) \geqq P\left(\hat{\Theta}_{2}^{\eta}>\exp (\beta(h(l-1)-\delta))\right) .
$$

Now, before $\hat{\Theta}_{2}^{\eta}, Z_{t}$ increases by 1 with rate smaller than $N^{2} e^{-\beta h}$ and decreases by 1 with rate greater than 1 . Comparing $Z_{t}$ with a birth and death process with these rates and using Lemma 1, the result follows.

Suppose now that $l>2 / h$ and choose $\varepsilon$ small enough so that

$$
2-h+\varepsilon<\min \{h(l-1)-\varepsilon, 2+h-\varepsilon\} .
$$

Let $\Omega_{2}$ be the event that $\check{\Theta}^{\eta}>e^{\beta(2-h+\varepsilon)}$ and before time $e^{\beta(2-h+\varepsilon)}$ the process $\left(\check{\sigma}_{t}^{\eta}\right)$ reaches a configuration in $\mathscr{R}$ in which the sides of the droplet of spins +1 are $l_{1}+a$ and $l_{2}+b$, with $a, b \geqq 0, a+b \geqq 1$. Now on the event $\left\{\check{\Theta}^{\eta}>e^{\beta(2-h+\varepsilon)}\right\}$ there is, from time 0 up to time $e^{\beta(2-h+\varepsilon)}$, at least one spin -1 outside $R$ which touches the droplet and is flipping in the process $\left(\check{\sigma}_{t}^{\eta}\right)$ with rate $e^{\beta(-2+h)}$. By considering the times

$$
u_{i}=i \exp (\beta(2-h+\varepsilon / 2))
$$

as in the proof of part (a) and using Lemma 2 we obtain

$$
\lim _{\beta \rightarrow \infty} P\left(\check{\Theta}^{\eta}>\exp (\beta(2-h+\varepsilon)),\left(\Omega_{2}\right)^{c}\right) \leqq \lim _{\beta \rightarrow \infty}\left(1-\left(1-\alpha_{+} / 2\right)^{\exp (\beta \varepsilon / 2)}\right)=0 .
$$

From Lemma 4, (9) and (8), we get

$$
\lim _{\beta \rightarrow \infty} P\left(\left(\Omega_{2}\right)^{c}\right)=0 .
$$

The proof of part (b) of the theorem is now easily completed using (7), (8) and the strong Markov property to restart each time a larger rectangular droplet is reached. Details are left again to the reader.

Now we describe the sets $\mathscr{A}, \mathscr{B}$ and $\mathscr{C}$ and sketch the proof of Proposition 2.

By Lemma 2 , if $0<h<2$, in a time of order 1 the system started from $\eta \in\{-1,+1\}^{\Lambda_{N}}$ can go with non-vanishing probability, as high as $\bar{\eta}$, but not higher. So $\mathscr{A}$ must be the set of configurations $\eta$ such that starting from $\bar{\eta}$ we are still likely to go to $-\underline{1}$ before $+\underline{1}$. But for any $\eta, \bar{\eta}$ is a configuration with the spins +1 forming rectangles, some of which may be degenerated into rings around the torus $\Lambda_{N}$. Also in $\bar{\eta}$, no spin -1 is neighbor of two of these rectangles. So we define 
$\mathscr{A}$ as the set of configurations $\eta$ such that the droplets of +1 spins in $\bar{\eta}$ are rectangles with longest sides not greater than $N-2$ and shortest sides smaller than $2 / h$; in case $0<h<1$ some of these droplets may also be rings of width 1 around the torus. Part (a) of Proposition 2 follows then easily from Theorem 1 and the techniques used to prove it.

In order to define $\mathscr{C}$ we need a new definition. Set $\eta^{\prime}=\bar{\xi}$, where $\xi=\underline{\eta}$. Again by Lemma 2 , if $0<h<2$, the system starting at $\eta$ can go with non-vanishing probability in a time of order 1 as low as $\underline{\eta}$, but not lower. But even if $\underline{\eta}$ is reached the system is likely to go to $\eta^{\prime}$ in a time of order 1 . So part (c) of Proposition 2 follows if we define $\mathscr{C}$ as the set of configurations $\eta$ such that at least one of the droplets of +1 spins in $\eta^{\prime}$ is a rectangle with all sides larger than $2 / h$ or a ring of width larger than 1 around the torus or, in case $1<h<2$, any ring around the torus.

Finally, $\mathscr{B}$ is the set of configurations not in $\mathscr{A}$ nor in $\mathscr{C}$ (for instance, those in $\mathscr{P}$ ). Starting from $\eta \in \mathscr{B}$ there is non-vanishing probability of reaching either $\bar{\eta}$ or $\eta^{\prime}$ in time of order 1 . Since $\eta$ is not in $\mathscr{A}, \bar{\eta}$ contains a droplet of +1 spins that is likely to grow. And since $\eta$ is not in $\mathscr{C}, \eta^{\prime}$ contains only small droplets which very likely disappear. So we can reach either $-\underline{1}$ or $+\underline{1}$ with non-vanishing probability, in the times given in the statement of the proposition.

\section{Metastable Behavior Around - 1}

In this section we prove Theorem 2.

Set $\mathscr{D}=\mathscr{A} U \mathscr{B}$ and define

$$
S=T-T(\mathscr{C})
$$

We will prove that

$$
\frac{T(\mathscr{C})}{\gamma_{\beta}} \rightarrow \tau \text { in distribution, }
$$

where $\tau$ is a unit mean exponential random variable and $\gamma_{\beta}$ is defined by

$$
P\left(T(\mathscr{C})>\gamma_{\beta}\right)=e^{-1} \text {. }
$$

Then we will prove

$$
\frac{S}{\gamma_{\beta}} \rightarrow 0 \text { in probability as } \beta \rightarrow \infty
$$

from what follows that

$$
\frac{T}{\gamma_{\beta}} \rightarrow \tau \text { in distribution as } \beta \rightarrow \infty \text {. }
$$

After doing this we need only to replace $\gamma_{\beta}$ by $E T$, which will be done using a standard argument.

To prove (10) we introduce a Glauber dynamics restricted to the set $\mathscr{D}$. Since we will need in the next section to restrict the Glauber dynamics to other sets we present the idea in a general fashion.

We say that a set $\mathscr{S}$ of configurations has the property of being connected if 
for any pair of configurations $\eta_{1}, \eta_{2} \in \mathscr{S}$ it is possible to go from $\eta_{1}$ to $\eta_{2}$ by a chain of transformations in which a single spin is flipped at each step without leaving $\mathscr{S}$. Observe that $\mathscr{D}$ is a connected set. The Glauber dynamics restricted to $\mathscr{S}$ is defined by the rates

$$
c_{\mathscr{S}}(x, \eta)= \begin{cases}c(x, \eta) & \text { if } \eta, \eta^{x} \in \mathscr{S} \\ 0 & \text { otherwise }\end{cases}
$$

Starting from $\eta \in \mathscr{S}$ the corresponding process $\left(\tilde{\sigma}_{t}^{\eta}, t \geqq 0\right)$ remains in $\mathscr{S}$ forever. It can be coupled with $\left(\sigma_{t}^{\eta}, t \geqq 0\right)$ in a very intuitive and useful way: the two processes jump together until the latter escapes from $\mathscr{S}$; at this moment the former process stays still and afterward they evolve independently. We will use this coupling several times in this section and in the next one so we give it the name of Coupling A. The following result is very easy to prove but crucial (see Proposition 5.10 of Chap. II in [Lig] for a discrete time version).

Lemma 5. If $\mathscr{S}$ is a connected set of configurations, then $\left(\tilde{\sigma}_{t}^{*}, t \geqq 0\right)$ is reversible with respect to its unique invariant probability measure, $\tilde{\mu}$ the Gibbs measured restricted to $\mathscr{S}$, defined by

$$
\tilde{\mu}(\eta)= \begin{cases}\frac{\mu(\eta)}{\sum_{\zeta \in \mathscr{S}} \mu(\zeta)} & \text { if } \eta \in \mathscr{S} \\ 0 & \text { otherwise. }\end{cases}
$$

In our case, with $\mathscr{S}=\mathscr{D}$, we have also

Lemma 6. In the standard case

a) for every $\eta \in \mathscr{D} \backslash\{-\underline{1}\}, H(\eta)>H(-\underline{1})$,

b) As $\beta \rightarrow \infty, \tilde{\mu}$ becomes concentrated on -1 , where $\tilde{\mu}$ is the Gibbs measure restricted to $\mathscr{D}$.

Proof. Part (b) clearly follows from part (a). To prove part (a) suppose first that $\eta=\eta^{\prime}$ (definition at the end of Sect. 3). Then all the clusters of spin +1 in $\eta$ are rectangles which have at least one side smaller than $2 / h$ or rings of width 1 around the torus and since $\eta \neq-1$ there is at least one such cluster. Now $H(\eta)-H(-1)$ is the sum of the contribution of each cluster of spins +1 , where for a rectangle $l_{1} \times l_{2}$ the contribution is $2\left(l_{1}+l_{2}\right)-l_{1} l_{2} h=f\left(l_{1}, l_{2}\right)$. But if we suppose, without loss of generality, that $l_{1} \leqq 2 / h$, then $f\left(l_{1}, l_{2}\right) \geqq 2 l_{2}>0$. The energy corresponding to a ring of width 1 is $N(2-h)>0$. So the proof is finished if $\eta=\eta^{\prime}$. On the other hand, if $\eta \neq \eta^{\prime}$, then $H(\eta)>H\left(\eta^{\prime}\right)$, because the transformations $\eta \rightarrow \zeta=\eta$ and $\zeta \rightarrow \eta^{\prime}=\bar{\zeta}$ strictly decrease the energy when they are not the identity and $0<\bar{\zeta}<2$. But then $H(\eta)-H(-1)>H\left(\eta^{\prime}\right)-H(-1) \geqq 0$, where the last inequality follows from the arguments given above, with the difference that now we may have $\eta^{\prime}=\underline{1}$.

We prove now (10) by showing the corresponding asymptotic loss of memory (a technique used in several of the former papers on the pathwise approach to metastability; see for instance [CGOV]). This consists in verifying that

$$
\lim _{\beta \rightarrow \infty} \Delta_{\beta}(s, t)=0 \text {, }
$$


where $\Delta_{\beta}(s, t)=\left|P\left(T(\mathscr{C})>(s+t) \gamma_{\beta}\right)-P\left(T(\mathscr{C})>s \gamma_{\beta}\right) P\left(T(\mathscr{C})>t \gamma_{\beta}\right)\right|$.

To see how (14) implies (10) see, for instance [CGOV].

Using the Markov property we have:

$$
P\left(T(\mathscr{C})>(s+t) \gamma_{\beta}\right)=\sum_{\eta \in \mathscr{D}} P\left(T(\mathscr{C})>s \gamma_{\beta}, \sigma_{s \gamma_{\beta}}^{-\frac{1}{2}}=\eta\right) P\left(T^{\eta}(\mathscr{C})>t \gamma_{\beta}\right)
$$

Hence by dividing this sum according to $\eta=-\underline{1}$ or $\eta \in \mathscr{D} \backslash\{-\underline{1}\}$ we have

$$
\Delta_{\beta}(s, t) \leqq P\left(T(\mathscr{C})>s \gamma_{\beta}, \sigma_{s \gamma_{\beta}}^{-1} \neq-\underline{1}\right) \leqq P\left(\tilde{\sigma}_{s \gamma_{\beta}^{-1}}^{-1} \neq-\underline{1}\right),
$$

where in the last inequality we used the Coupling A with $\mathscr{S}=\mathscr{D}$. The right-hand side of (15) can be controlled using monotonicity, but we avoid its use because we will need a similar estimate in the next section where monotonicity is not available.

We now couple $\left(\tilde{\sigma}_{t}^{\tilde{\mu}}, t \geqq 0\right)$ and $\left(\tilde{\sigma}_{t}^{-1}, t \geqq 0\right)$ in the following manner: the initial configuration of the former one is chosen with respect to $\tilde{\mu}$; they evolve independently until they meet and after that they evolve together. This is a very standard sort of coupling and here we call it Coupling B. So

$$
P\left(\tilde{\sigma}_{s \gamma_{\beta}}^{-1} \neq-\underline{1}\right) \leqq P\left(\tilde{\sigma}_{0}^{-1} \neq \tilde{\sigma}_{0}^{\tilde{\mu}}\right)+P\left(\tilde{\sigma}_{s \gamma_{\beta}}^{\tilde{\mu}} \neq-\underline{1}\right) \leqq 2 \tilde{\mu}(\mathscr{D} \backslash\{-\underline{1}\}),
$$

where the last inequality follows from the invariance of $\tilde{\mu}$ (Lemma 5). Now (15), (16) and Lemma 6, imply (14) and therefore (10). To prove (12) we observe that since $T$ is larger than the time needed for the first spin to flip on $-\underline{1}$, we have

$$
\gamma_{\beta} \geqq \exp (\beta(4-h)) \text {. }
$$

(12) follows now using part (b) of Proposition 2, finishing the proof of (13). To replace $\gamma_{\beta}$ by $E T$, observe that, by monotonicity,

$$
P\left(T>\gamma_{\beta} u\right) \leqq\left(P\left(T>\gamma_{\beta}\right)\right)^{\lfloor u\rfloor} .
$$

Using (13) it follows that $P\left(T>\gamma_{\beta}\right)<1$ if $\beta$ is large, so we can use dominated convergence below:

$$
\lim _{\beta \rightarrow \infty} \frac{E T}{\gamma_{\beta}}=\lim _{\beta \rightarrow \infty} \frac{\int_{0}^{\infty} P(T>t) d t}{\gamma_{\beta}}=\lim _{\beta \rightarrow \infty} \int_{0}^{\infty} P\left(T / \gamma_{\beta}>u\right) d u=\int_{0}^{\infty} e^{-u} d u=1 .
$$

And the proof of part (c) of Theorem 2 is finished.

We turn now to the proof of part (b) of Theorem 2. At a certain point below we will have to use part (b) of Theorem 3, which will be proved in the next section using part (a) of Theorem 2, proved above, but there is no circularity in the reasoning.

Given sets of configurations $\mathscr{S}_{1}, \mathscr{S}_{2}, \ldots$, define

$$
F_{m}\left(\mathscr{S}_{1}, \ldots, \mathscr{S}_{m}\right)= \begin{cases}1 & \text { if }-\underline{1} \in \mathscr{S}_{i}, \quad i=1, \ldots, m \\ 0 & \text { otherwise }\end{cases}
$$

and

$$
G_{m}\left(\mathscr{S}_{1}, \ldots, \mathscr{S}_{m}\right)=F_{m}\left(-\mathscr{S}_{1}, \ldots,-\mathscr{S}_{m}\right),
$$

where $-\mathscr{S}=\{-\eta: \eta \in \mathscr{S}\}$. We set also $F_{0}=G_{0}=1$. 
We have to prove that for every positive integer $k$ and every $0<t_{1}<\cdots<t_{k}<\infty$,

$$
\begin{aligned}
\lim _{\beta \rightarrow \infty} P\left(\sigma_{t_{k} E T}^{-\frac{1}{E} \in \mathscr{S}_{i}}, i=1, \ldots, k\right) \\
\quad=\sum_{m=0}^{k} P\left(t_{m}<\tau<t_{m+1}\right) F_{m}\left(\mathscr{S}_{1}, \ldots, \mathscr{S}_{m}\right) G_{k-m}\left(\mathscr{S}_{m+1}, \ldots, \mathscr{S}_{k}\right),
\end{aligned}
$$

where $t_{0}=0, t_{k+1}=\infty$ and $\tau$ is a unit mean exponential random variable.

To prove (18) we write $u_{i}=t_{i} E T$ and

$$
P\left(\sigma_{u_{i}}^{-1} \in \mathscr{S}_{i}, i=1, \ldots, k\right)=\sum_{\{0 \leqq m \leqq k, m \leqq n \leqq k\}} P\left(\sigma_{u_{i}}^{-1} \in \mathscr{S}_{i}, i=1, \ldots, k \mid E_{m, n}\right) P\left(E_{m, n}\right),
$$

where $E_{m, n}=\left\{u_{m} \leqq T(\mathscr{C})<u_{m+1}, u_{n} \leqq T<u_{n+1}\right\}$.

From part (a) of Theorem 2, (10) and (17) we have that as $\beta \rightarrow \infty, T / E T \rightarrow \tau$ in distribution and $(T-T(\mathscr{C})) / E T \rightarrow 0$ in probability. Hence

$$
\lim _{\beta \rightarrow \infty} P\left(E_{m, n}\right)=\left\{\begin{array}{lll}
0 & \text { if } & n \neq m \\
P\left(t_{m}<\tau<t_{m+1}\right) & \text { if } & n=m
\end{array} .\right.
$$

In particular in the limit, we can neglect in (19) all the terms with $n \neq m$.

But using Coupling A

$$
P\left(\sigma_{u_{i}}^{-} \in \mathscr{T}_{i}, i=1, \ldots, m \mid E_{m, n}\right)=P\left(\tilde{\sigma}_{u_{i}}^{-} \in \mathscr{T}_{i}, i=1, \ldots, m \mid E_{m, n}\right) .
$$

Without conditioning we obtain, as in (16),

$$
\lim _{\beta \rightarrow \infty} P\left(\tilde{\sigma}_{u_{i}}^{-1} \in \mathscr{S}_{i}, i=1, \ldots, m\right)=F_{m}\left(\mathscr{S}_{1}, \ldots, \mathscr{S}_{m}\right)
$$

And since the limit in (20) is positive in the case $n=m$ and $F_{m}\left(\mathscr{S}_{1}, \ldots, \mathscr{S}_{m}\right)=0$ or 1 :

$$
\lim _{\beta \rightarrow \infty} P\left(\sigma_{u_{i}}^{-1} \in \mathscr{S}_{i}, i=1, \ldots, m \mid E_{m, m}\right)=F_{m}\left(\mathscr{S}_{1}, \ldots, \mathscr{S}_{m}\right) .
$$

To control what happens after $T$, we compare the Glauber dynamics with different values of the external field. When the external field has a value which is different from the one that appears in the statement of the theorem, we write it explicitly. It is known (see for instance Theorem 1.5 of Chap. III of [Lig]) that we can couple two Glauber dynamics with external fields $h_{1}<h_{2}$ in such a way that

$$
\sigma_{h_{1}, t}^{-\frac{1}{1}} \leqq \sigma_{h_{2}, t}^{-1}
$$

for every $t \geqq 0$.

We want to show that

$$
\lim _{\beta \rightarrow \infty} P\left(\sigma_{u_{i}}^{-1} \in \mathscr{S}_{i}, i=m+1, \ldots, k \mid E_{m, m}\right)=G_{k-m}\left(\mathscr{S}_{m+1}, \ldots, \mathscr{S}_{k}\right) .
$$

This will follow from the Markov property once we show that for every positive integer $j$ and $0<s_{1}<\cdots<s_{j}<\infty$,

$$
\lim _{\beta \rightarrow \infty} P\left(\sigma_{v_{i}}^{+1} \in \mathscr{S}_{i}, i=1, \ldots, j\right)=G_{j}\left(\mathscr{S}_{1}, \ldots, \mathscr{S}_{j}\right),
$$

where $v_{i}=s_{i} E T$. Using symmetry

$$
P\left(\sigma_{v_{i}}^{+1} \in \mathscr{S}_{i}, i=1, \ldots, j\right)=P\left(\sigma_{-\bar{h}, v_{i}}^{-1} \in-\mathscr{S}_{i}, i=1, \ldots, j\right) .
$$


Therefore (25) will follow using (23) if we prove that for some $h^{\prime}>-h$,

$$
\lim _{\beta \rightarrow \infty} P\left(\sigma_{h^{\prime}, v_{i}}^{-1} \in-\mathscr{S}_{i}, i=1, \ldots, j\right)=F_{j}\left(-\mathscr{S}_{1}, \ldots,-\mathscr{S}_{j}\right)=G_{j}\left(\mathscr{S}_{1}, \ldots, \mathscr{S}_{j}\right) .
$$

Set

$$
T^{\prime}=\inf \left\{t \geqq 0: \sigma_{h^{\prime}, t}^{-\frac{1}{t}}=+\underline{1}\right\} .
$$

We can choose $h^{\prime}<h$ close enough to $h$ so that $\left\lceil 2 / h^{\prime}\right\rceil=\lceil 2 / h\rceil$. Then we can apply part (b) of Theorem 3 to conclude that

$$
\frac{\log \left(E T / E T^{\prime}\right)}{\beta}=\Gamma(h)-\Gamma\left(h^{\prime}\right)<0 .
$$

Hence, $E T / E T^{\prime} \rightarrow 0$ as $\beta \rightarrow \infty$. Using part (a) of Theorem 2 in the case in which the field is $h^{\prime}$ we have then

$$
\lim _{\beta \rightarrow \infty} P\left(v_{j}<T^{\prime}\right)=\lim _{\beta \rightarrow \infty} P\left(s_{j}<\left(T^{\prime} / E T^{\prime}\right)\left(E T^{\prime} / E T\right)\right)=1 .
$$

Now, from the same arguments which led to (21), we obtain (26), and hence (25) and (24). Equation (18) follows from (19), (20), (22) and (24) finishing the proof of Theorem 2.

\section{Escape from the Metastable Situation}

We will prove in this section Theorem 3. Our first goal is to show that for every $\varepsilon>0$,

$$
\lim _{\beta \rightarrow \infty} P(T>\exp (\beta(\Gamma(h)+3 \varepsilon)))=0 .
$$

The main estimate in this direction is

Lemma 7. In the standard case, for every $\varepsilon>0$, there is $\beta_{0}<\infty$ such that for $\beta>\beta_{0}$,

$$
P\left(T(\mathscr{C}) \leqq \exp (\beta((L-1) h+2 \varepsilon)) \geqq \frac{1}{3} \exp (-\beta e(h, L)),\right.
$$

where $e(h, l)$ was defined in (2).

Proof. Let $\mathscr{E}$ be the set of configurations in which all spins +1 are inside a square $L \times L$ (but there may be also -1 spins in this square). Set $\mathscr{F}=\mathscr{D} U \mathscr{E}$ and consider the Glauber dynamics restricted to the set $\mathscr{F}$ in the fashion described in the last section. Since $\mathscr{F}$ is a connected set, Lemma 5 applies. As in the last section we will use the notation $\left(\tilde{\sigma}_{t}\right)$ and $\tilde{\mu}$ for the restricted process and corresponding Gibbs measure without making $\mathscr{F}$ explicit. Later we will use other sets to restrict the dynamics and will use again the same notation, but no confusion should arise.

Define

$$
\tilde{T}(\mathscr{C})=\inf \left\{t \geqq 0: \sigma_{t}^{-1} \in \mathscr{C}\right\} .
$$

Now using the coupling A, and writing $V=\exp (\beta((L-1) h+2 \varepsilon))$, we have

$$
P(T(\mathscr{C})>V) \leqq P(\widetilde{T}(\mathscr{C})>V)
$$

We couple now $\left(\sigma_{t}^{-1}\right)$ to $\left(\tilde{\sigma}_{t}^{\tilde{\mu}}\right)$ so that they evolve independently until they meet 
and from then on evolve together (Coupling B). Now

$$
P(\tilde{T}(\mathscr{C})>V) \leqq P\left(\sigma_{t}^{-1} \neq \tilde{\sigma}_{t}^{\tilde{\mu}} \text { for } 0 \leqq t \leqq V\right)+P\left(\tilde{\sigma}_{V}^{\tilde{\mu}} \notin \mathscr{C}\right) .
$$

Write $W=\exp (\beta((L-1) h+\varepsilon))$. Then using the Markov property,

$$
P\left(\left(\tilde{\sigma}_{t}^{-1} \neq \tilde{\sigma}_{t}^{\tilde{\mu}} \text { for } 0 \leqq t \leqq V\right) \leqq\left(\sup _{\eta, \zeta \in \mathscr{F}} P\left(\tilde{\sigma}_{t}^{\eta} \neq \tilde{\sigma}_{t}^{\zeta} \text { for } 0 \leqq t \leqq W\right)\right)^{\lfloor\operatorname{lexp}(\beta \varepsilon)]},\right.
$$

where $\left(\tilde{\sigma}_{t}^{\eta}\right)$ and $\left(\tilde{\sigma}_{t}^{\zeta}\right)$ are independent processes. The next step will characterize $\exp (\beta(L-1) h)$ as the relaxation time for the process $\left(\tilde{\sigma}_{t}\right)$. In a time of this order the process is likely to go to -1 , where it stays for an exponential time of mean $\exp (\beta(4-h))$, much greater (at low temperatures) than $\exp (\beta(L-1) h)$ before a spin -1 appears. (Since $0<h<2$, we have $(L-1) h<4-h$.) More precisely we claim that

$$
\lim _{\beta \rightarrow \infty} \sup _{\eta \in \mathscr{F}} P\left(\tilde{\sigma}_{t}^{\eta} \neq-\underline{1} \text { for } 0 \leqq t \leqq W\right)=0 .
$$

And then, from the remark above (if $\varepsilon$ is small)

$$
\lim _{\beta \rightarrow \infty} \sup _{\eta \in \mathscr{F}} P\left(\tilde{\sigma}_{W}^{\eta} \neq-1\right)=0
$$

so that

$$
\lim _{\beta \rightarrow \infty} \sup _{\eta, \zeta \in \mathscr{F}} P\left(\tilde{\sigma}_{t}^{\eta} \neq \tilde{\sigma}_{t}^{\zeta} \text { for } 0 \leqq t \leqq W\right)=0 .
$$

The proof of the crucial relation (31) is similar to that of part (a) of Theorem 1, done in Sect. 3, so we merely sketch it, leaving the details for the reader. First observe that in the dynamics restricted to $\mathscr{F}$, in any interval of time of order 1 there is a positive probability (uniform in $\beta$ and the initial configuration) that the system will reach a configuration where all the clusters of spin +1 are rectangles and no spin -1 has two neighbors which are +1 . This configuration must be in $\mathscr{A} U \mathscr{G}$ ( $\mathscr{G}$ is the set of configurations with a single critical droplet). So in time of order 1 the system is likely to reach such a configuration. If it is in $\mathscr{A}$, then part (a) of Proposition 2 completes the proof of (31) since $(L-1) h>2-h$. If the system is in $\mathscr{G}$, then no spin -1 outside the square $L \times L$ can flip in the dynamics restricted to $\mathscr{F}$ until the system reaches $\mathscr{D}$. But the "corner erosion mechanism" will then make the system hit $\mathscr{D}$ with large probability in a time $\exp (\beta((L-1) h+\varepsilon / 2))($ as in Lemma 3). But each time the system reaches $\mathscr{D}$ it has positive probability of reaching $\mathscr{A}$ in a time of order 1 (Lemma 2 ) and then -1 in a time $\exp (\beta(L-1) h)$. This completes the sketch of the proof of (31) and hence of (32).

Using now (28), (29), (30) and (32), we have for $\beta$ larger than $\beta_{1}<\infty$,

$$
P(T(\mathscr{C})>V) \leqq(1 / 2)^{[\exp (\beta \varepsilon)]}+(1-\tilde{\mu}(\mathscr{C})) .
$$

Hence

$$
P(T(\mathscr{C}) \leqq V) \geqq \tilde{\mu}(\mathscr{G})-(1 / 2)^{\mid \exp (\beta \varepsilon) !} .
$$

For any $\eta \in \mathscr{F} \backslash\{-\underline{1}\}$ we have

$$
H(\eta)-H(-\underline{1})>0
$$


because if $\eta \in \mathscr{D} \backslash\{-\underline{1}\}$ this is Lemma 6 and if $\eta \in \mathscr{F} \backslash \mathscr{D}$ then $\bar{\eta} \in \mathscr{G}$ and therefore

$$
H(\eta)-H(-\underline{1}) \geqq H(\bar{\eta})-H(-\underline{1})=e(h, L)>0 .
$$

So, for $\beta$ larger than some $\beta_{2}<\infty$,

$$
\tilde{\mu}(\mathscr{G})=\sum_{\sigma \in \mathscr{G}} \exp (-\beta H(\sigma)) / \sum_{\sigma \in \mathscr{F}} \exp (-\beta H(\sigma)) \geqq \frac{1}{2} \exp (-\beta e(h, L)) .
$$

Lemma 7 follows from (33) and (34).

To prove (27) now write

$$
\begin{aligned}
& P(T>\exp (\beta(\Gamma(h)+3 \varepsilon))) \\
& \leqq P(T>\exp (\beta(\Gamma(h)+3 \varepsilon)), T(\mathscr{C})<\exp (\beta(\Gamma(h)+3 \varepsilon))-\exp (\beta((L-1) h+2 \varepsilon))) \\
& \quad+P(T(\mathscr{C})>\exp (\beta(\Gamma(h)+3 \varepsilon))-\exp (\beta((L-1) h+2 \varepsilon))) .
\end{aligned}
$$

The first term in the right-hand side of (35) goes to zero by part (b) of Proposition 2 since $h(L-1)>2-h$ as we have $L>2 / h$. For the second term consider the times

$$
t_{i}=i \exp (\beta((L-1) h+2 \varepsilon)), \quad i=1,2, \ldots
$$

and use monotonicity to obtain

$$
\begin{aligned}
& P(T(\mathscr{C})>\exp (\beta(\Gamma(h)+3 \varepsilon))-\exp (\beta((L-1) h+2 \varepsilon))) \\
& \quad \leqq[P(T(\mathscr{C})>\exp (\beta((L-1) h+2 \varepsilon)))]^{\exp (\beta(e(h, L)+\varepsilon))-2}
\end{aligned}
$$

that also goes to zero, by Lemma 7 .

We will prove now part (c) of Theorem 3. Define $|\eta|=\left|\left\{x \in \Lambda_{N}: \eta(x)=1\right\}\right|$ and

$$
\begin{aligned}
\mathscr{H} & =\left\{\eta \in\{-1,+1\}^{\Lambda_{N}}:|\eta| \leqq L^{2}\right\}, \\
\mathscr{I}_{s} & =\left\{\eta \in\{-1,+1\}^{\Lambda_{N}}:|\eta|=s\right\}, \\
\mathscr{I} & =\mathscr{I}_{L^{2}},
\end{aligned}
$$

and observe that $\mathscr{G} \subset \mathscr{I} \subset \mathscr{H}$.

Lemma 8. In the standard case,

a) for every $\eta \in \mathscr{H} \backslash\{-\underline{1}\}, H(\eta)>H(-\underline{1})$,

b) for every $\eta \in \mathscr{I} \backslash \mathscr{G}, H(\eta)-H(-\underline{1}) \geqq e(h, L)+2$.

Proof. If we transform a configuration $\eta$ with more than one cluster of spins +1 into a configuration $\zeta$ with a single cluster by translating each cluster until it touches another one (without deforming it), clearly $H(\eta) \geqq H(\zeta)$. So the energy must be minimized over $\mathscr{I}_{s}$ at configurations with a unique cluster of spins +1 . For such a configuration $\eta \in \mathscr{I}_{s}$, with $0<s \leqq L^{2}$, this cluster can not "go around the torus $\Lambda_{N}$," since $N>N(h)>L^{2}$. (This is the only place in the whole paper where we use this hypothesis contained in the definition of the standard case.) Let now $M$ and $N$ be the sides of the smallest rectangle that contains the cluster of spins +1 . Then a simple argument shows that the boundary of the cluster must have length at least $2(M+N)$ and so

$$
H(\eta) \geqq-h s+2(M+N) .
$$


But $M N \geqq s$ and $M, N>0$ imply that $M+N \geqq 2 \sqrt{s}$. So

$$
H(\eta) \geqq-h s+4 \sqrt{s}=e(h, \sqrt{s}) .
$$

Part (a) of the lemma follows now from the fact that $\mathscr{H} \backslash\{-\underline{1}\}=\bigcup_{s=1}^{L^{2}} \mathscr{I}_{s}$ and, since $0<h<2$, for $0<\sqrt{s} \leqq L<4 / h$ we have $e(h, \sqrt{s})>0$.

For part (b) of the lemma we proceed as above and observe that, since $M N \geqq L^{2}, M, N \in\{1,2, \ldots\}$ and at least one between $M$ and $N$ is not $L$ (because we are not in $\mathscr{G}$ ) we must have $M+N \geqq 2 L+1$. The conclusion follows from (37).

The following lemma will be used several times. $\tilde{T}(\eta)$ below is the hitting time of $\eta$ starting from -1 for the Glauber dynamics restricted to $\mathscr{S}$.

Lemma 9. Let $\mathscr{S}$ be a connected set of configurations containing $-\underline{1}$. Suppose that for every $\eta \in \mathscr{S} \backslash\{-\underline{1}\}, H(\eta)>H(-1)$. Then for every $\eta \in \mathscr{S}$ and every $\varepsilon>0$,

$$
\lim _{\beta \rightarrow \infty} P(\tilde{T}(\eta)<\exp (\beta(H(\eta)-H(-\underline{1})-\varepsilon)))=0 .
$$

Proof. The argument is the same used to prove part of Lemma 1, and is borrowed from [LS]. Couple $\left(\sigma_{t}^{-\underline{1}}\right)$ to $\left(\tilde{\sigma}_{t}^{\tilde{\mu}}\right)$ so that they evolve independently until they meet, evolving together after this (Coupling B). Now

$$
\begin{aligned}
P(\tilde{T}(\eta) & <\exp (\beta(H(\eta)-H(-\underline{1})-\varepsilon))) \leqq P\left(\tilde{\sigma}_{0}^{-1} \neq \tilde{\sigma}_{0}^{\tilde{\mu}}\right) \\
& +P\left(\tilde{\sigma}_{t}^{\tilde{\mu}}=\eta \text { for some } t \in\left\{\beta^{-1}, 2 \beta^{-1}, \ldots,\lceil\beta \exp (\beta(H(\eta)-H(-1)-\varepsilon))\rceil \beta^{-1}\right\}\right) \\
& +P\left(\tilde{\sigma}_{t}^{\tilde{\mu}} \text { changes state between times } \tilde{T}^{\tilde{\mu}}(\eta) \text { and } \tilde{T}^{\tilde{\mu}}(\eta)+\beta^{-1}\right) \\
\leqq & \tilde{\mu}(\mathscr{S} \backslash\{-\underline{1}\})+(\beta \exp (\beta(H(\eta)-H(-\underline{1})-\varepsilon))+1) \tilde{\mu}(\eta) \\
& +\left(1-\exp \left(-\beta^{-1} N^{2}\right)\right),
\end{aligned}
$$

which goes to 0 as $\beta \rightarrow \infty$.

Consider the Glauber dynamics $\left(\tilde{\sigma}_{t}\right)$ restricted to $\mathscr{H}$. Since only one spin can flip at a time in the Glauber dynamics, for every path we must have $\widetilde{T}(\mathscr{I})=T(\mathscr{I})<T$, where $\left(\tilde{\sigma}_{t}^{-1}\right)$ and $\left(\sigma_{t}^{-1}\right)$ are coupled in the usual fashion (Coupling $A$ ). So

$$
\begin{aligned}
P(T(\mathscr{G})>T) & =P(T(\mathscr{I})<T<T(\mathscr{G})) \leqq P(\tilde{T}(\mathscr{I} \backslash \mathscr{G})<T) \\
& \leqq P(\tilde{T}(\mathscr{I} \backslash \mathscr{G})<\exp (\beta(\Gamma(h)+\varepsilon)))+P(T>\exp (\beta(\Gamma(h)+\varepsilon)))
\end{aligned}
$$

for arbitrary $\varepsilon>0$. But we can choose $\varepsilon$ so that $\Gamma(h)+\varepsilon=e(h, L)+(L-1) h+\varepsilon<$ $e(h, L)+2 \leqq H(\eta)$ for every $\eta \in \mathscr{I} \backslash \mathscr{G}$, by Lemma 8 . Part (c) of Theorem 3 is now a consequence of (39), (27) and Lemma 9.

Define now $\mathscr{B}_{0}$ as the set of configurations $\eta \in \mathscr{A}^{c}$ which can be obtained by taking a configuration in $\mathscr{A}$ and flipping one of its spins -1 .

Lemma 10. In the standard case
a) $\mathscr{B}_{0} \subset \mathscr{B}$,
b) $P\left(T(\mathscr{B})=T\left(\mathscr{B}_{0}\right)\right)=1$
c) $P(T(\mathscr{B})<T(\mathscr{C}))=P\left(T\left(\mathscr{B}_{0}\right)<T(\mathscr{C})\right)=1$.

Proof. Take $\eta \in \mathscr{B}_{0}, \zeta \in \mathscr{A}$ and $x \in \Lambda_{N}$ such that $\zeta(y)=\eta(y)$ for $y \neq x, \xi(x)=-1$, and $\eta(x)=+1$. Since $\zeta \in \mathscr{A}$ and $\eta \in \mathscr{A}^{c}$, we must have $\bar{\zeta} \in \mathscr{A}$ and $\bar{\eta} \in \mathscr{C}$. In particular $\bar{\zeta} \neq \bar{\eta}$. 
Therefore in $\zeta$ and in $\eta, x$ can have at most one neighboring spin that is +1 . But this implies that $\eta(x)=-1$, and so $\eta=\zeta$, implying that $\eta \in \mathscr{A}$. Since $\bar{\eta} \in \mathscr{C}$ and $\eta \in \mathscr{A}$, we must have $\eta \in \mathscr{B}$ and part (a) is proven. Parts (b) and (c) follow from part (a), and the fact that only one spin flips at a time in the Glauber dynamics.

Set now

$$
\begin{aligned}
\mathscr{P}_{1} & =\left\{\eta \in \mathscr{B}_{0}: \bar{\eta} \in \mathscr{G}\right\}, \\
\mathscr{S}_{1} & =\left\{\eta \in \mathscr{B}_{0} \backslash \mathscr{P}_{1}:|\eta|<L^{2}\right\}, \\
\mathscr{S}_{2} & =\left\{\eta \in \mathscr{B}_{0} \backslash \mathscr{P}_{1}:|\eta| \geqq L^{2}\right\} .
\end{aligned}
$$

Lemma 11. In the standard case,

$$
\lim _{\beta \rightarrow \infty} P\left(T\left(\mathscr{P}_{1}\right)>T(\mathscr{C})\right)=0 .
$$

Proof. From part (c) of Lemma 10,

$$
P\left(T\left(\mathscr{P}_{1}\right)>T(\mathscr{C})\right) \leqq P\left(T\left(\mathscr{B}_{0} \backslash P_{1}\right)<T(\mathscr{C}) \leqq T(\mathscr{G})\right) .
$$

Since only one spin flips at a time,

$$
P\left(T\left(\mathscr{S}_{2}\right)<T(\mathscr{G})\right) \leqq P(T(\mathscr{I} \backslash \mathscr{G})<T(\mathscr{G})) .
$$

Observe that if the process is in $\eta \in \mathscr{S}_{1}$ and it goes to $\bar{\eta}$ flipping only spins from -1 to +1 , then it must hit $\mathscr{I}$ before it hits $\mathscr{G}$. Hence, from Lemma 2 and the strong Markov property

$$
P(T(\mathscr{I} \backslash \mathscr{G}))<T(\mathscr{G})) \geqq \alpha_{+} P\left(T\left(S_{1}\right)<T(\mathscr{G})\right)
$$

using (41), (42), (43) and the last inequality in (39),

$$
\begin{aligned}
\lim _{\beta \rightarrow \infty} P\left(T\left(\mathscr{P}_{1}\right)>T(\mathscr{C})\right) & \leqq \lim _{\beta \rightarrow \infty}\left[\left(1+\frac{1}{\alpha_{+}}\right) P(T(\mathscr{I} \backslash \mathscr{G})<T(\mathscr{G}))\right] \\
& \leqq \lim _{\beta \rightarrow \infty}\left[\left(1+\frac{1}{\alpha_{+}}\right) P(\tilde{T}(\mathscr{I} \backslash \mathscr{G})<T)\right]=0 .
\end{aligned}
$$

Lemma 12. In the standard case

a) $\mathscr{P}=\left\{\eta \in \mathscr{P}_{1}:|\eta| \geqq L^{2}-L+1\right\}$,

b) For every $\eta \in \mathscr{P}_{1} \backslash \mathscr{P}, H(\eta)-H(-\underline{1})>\Gamma(h)$.

Proof. Clearly $\mathscr{P}$ is contained in the set in the right-hand side of (a). We will prove now the complementary relation. If $\eta \in \mathscr{P}_{1}$ there is $\xi \in \mathscr{A}$ such that $\eta$ and $\xi$ differ only at one site, say $x$. Let $R$ be the square where $\bar{\eta}$ has the spins +1 , that we take, without loss of generality, to be given by $\{1, \ldots, L\}^{2}$. We use now an idea from percolation theory (see [Kes]), namely the duality between site percolation and ${ }^{*}$-site percolation. The ${ }^{*}$-neighbors of a site $x \in \Lambda_{N}$ are the 8 sites $y$ such that $\max \left\{\left|y_{1}-x_{1}\right|,\left|y_{2}-x_{2}\right|\right\}=1$. A chain (respectively *-chain) of sites from $A \subset \Lambda_{N}$ to $B \subset \Lambda_{N}$ is a sequence $x_{1}, x_{2}, \ldots, x_{n}$ such that $x_{1} \in A, x_{n} \in B$ and $x_{i}$ and $x_{i-1}$ are neighbors (respectively $*$-neighbors), $i=1, \ldots, n-1$. Consider the four external slices of $R: H_{1}=\{1, \ldots, L\} \times\{1\}, H_{L}=\{1, \ldots, L\} \times\{L\}, V_{1}=\{1\} \times\{1, \ldots, L\}$ and $V_{L}=\{L\} \times\{1, \ldots, L\}$. A vertical path (respectively *-path) in $R$ is a chain (respectively *-chain) from $H_{1}$ to $H_{L}$ contained in $R$. The definition of a horizontal path is analogous with $V_{1}$ and $V_{L}$ replacing $H_{1}$ and $H_{L}$. By duality, either there 
is a horizontal (respectively vertical) path where all the spins are +1 or there is a vertical (respectively horizontal) *-path where all the spins are -1 . It is easy to see that in $\xi$ we can not have both a horizontal and a vertical *-path in $R$ where all the spins are +1 since otherwise we would have $\xi \notin \mathscr{A}$. Hence we must have in $\xi$ a horizontal or a vertical path in $R$ where all the spins are -1 . Since we are assuming that $|\eta| \geqq L^{2}-L+1$, we must have $|\xi| \geqq L^{2}-L$ and the only way for this to happen is that the chain of spins -1 is a straight line. But since $\xi \in \mathscr{A}$, this straight line must be one of the external slices of $R$. Hence $\xi$ consists in one rectangle $L \times(L-1)$ of spins +1 in a sea of spins -1 and $\eta \in \mathscr{P}$.

To prove part (b) we write $H(\eta)=H_{1}(\eta)+H_{2}(\eta)$, where

$$
H_{1}(\eta)=-\frac{1}{2} \sum_{\langle x, y\rangle} \eta(x) \eta(y), \quad H_{2}(\eta)=-\frac{h}{2} \sum_{x} \eta(x) .
$$

It is clear that $H_{1}(\bar{\eta}) \leqq H_{1}(\eta)$ for every configuration $\eta$. If $\eta \in \mathscr{P}_{1} \backslash \mathscr{P}$, then $\bar{\eta} \in \mathscr{G}$ and from part (a) of the lemma

$$
\begin{aligned}
H(\eta) & \geqq H_{1}(\bar{\eta})+H_{2}(\eta) \geqq H(\bar{\eta})+h L \\
& =H(-\underline{1})+e(h, L)+h L \geqq H(-\underline{1})+\Gamma(h)+h .
\end{aligned}
$$

Considering now the Glauber dynamics restricted to $\mathscr{D}$ coupled to the Glauber dynamics by coupling $A$ and using Lemma 11 we have

$$
\begin{aligned}
\lim _{\beta \rightarrow \infty} P(T(\mathscr{P})>T) \leqq & \lim _{\beta \rightarrow \infty} P\left(T\left(\mathscr{P}_{1}\right)<T(\mathscr{C}) \leqq T<T(\mathscr{P})\right) \\
\leqq & \lim _{\beta \rightarrow \infty} P\left(\widetilde{T}\left(\mathscr{P}_{1} \backslash \mathscr{P}\right)<T\right) \leqq \lim _{\beta \rightarrow \infty}\left[P\left(\widetilde{T}\left(\mathscr{P}_{1} \backslash \mathscr{P}\right)<\exp (\beta(\Gamma(h)+\varepsilon))\right)\right. \\
& +P(T>\exp (\beta(\Gamma(h)+\varepsilon)))]=0
\end{aligned}
$$

where $\varepsilon>0$ was chosen conveniently and we used (27), Lemma 9, part (a) of Lemma 6 and part (b) of Lemma 12. This completes the proof of part (d) of Theorem 3. It is easy now to complete the proof of part (a). Using (45) and the same coupling and arguments given there, for $\varepsilon>0$

$$
\begin{aligned}
\lim _{\beta \rightarrow \infty} P(T<\exp (\beta(\Gamma(h)-\varepsilon))) & \leqq \lim _{\beta \rightarrow \infty} P(T(\mathscr{P})<T(\mathscr{C})<\exp (\beta(\Gamma(h)-\varepsilon))) \\
& \leqq \lim _{\beta \rightarrow \infty} P(\tilde{T}(\mathscr{P})<\exp (\beta(\Gamma(h)-\varepsilon))=0 .
\end{aligned}
$$

Part (a) of Theorem 3 follows from (27) and (46). Part (b) of Theorem 3 follows easily from part (a) and part (a) of Theorem 2 . This completes the proof of Theorem 1.

We end this section by observing that in fact our proof of Theorem 3 gives us even more information about the way the system escapes from the metastable situation around $-\underline{1}$. It follows that

$$
\lim _{\beta \rightarrow \infty} P\left(T\left(\mathscr{A}^{c}\right)=T(\mathscr{P})\right)=1,
$$

i.e., the system is likely to escape from $\mathscr{A}$ jumping to $\mathscr{P}$. But once in $\mathscr{P}$ there are probabilities of order 1 that in a time of order 1 the system either jumps back to $\mathscr{A}$ (loosing the protuberance of the droplet) or jumps to $\mathscr{C}$ (if the protuberance is enlarged and stabilized by the appearance of another spin +1 neighbor to the original one). The probability of anything else happening with the configuration in $\mathscr{P}$ vanishes as $\beta \rightarrow \infty$. If the portuberance is lost and the system is back to $\mathscr{A}$, 
Then it is likely to return to $-\underline{1}$ before reentering $\mathscr{A}^{c}$ and everything restarts anew. On the other hand, if the system enters in $\mathscr{C}$, then it is likely to go to $+\underline{1}$ before leaving $\mathscr{C}$ again. So we see that the system will probably visit $\mathscr{P}$ a finite number of times returning to $-\underline{1}$ afterward except for the last time when it goes to $+\underline{1}$ and in its way to $+\underline{1}$ it will very likely through $\mathscr{G}$.

Acknowledgements. Continuous and helpful discussions on metastability with the following people are thankfully acknowledged: M. Aizenman, M. Cassandro, A. Galves, J. Lebowitz, J. F. Perez, E. Presutti, E. Olivieri, M. E. Vares.

\section{References}

[Bra] Brassesco, S.: Tunelling for a non-linear heat equation with noise. Preprint (1989)

[CGOV] Cassandro, M., Galves, A., Olivieri, E., Vares, M. E.: Metastable behavior of stochastic dynamics: A pathwise approach. J. Stat. Phys. 35, 603-634 (1984)

[COP] Cassandro, M. Olivieri, E., Picco, P.: Small random perturbations of infinite dimensional dynamical systems and nucleation theory. Ann. Inst. Henri Poincaré (Phys. Theor.) 44, 343-396 (1986)

[GOV] Galves, A., Olivieri, E., Vares, M. E.: Metastability for a class of dynamical systems subject to small random perturbations. Ann. Probab. 15, 1288-1305 (1987)

[EGJL] Eston, V. R., Galves, A., Jacobi, C. M., Langevin, R.: Dominance switch between two interacting species and metastability. Preprint (1988)

[HMM] Huiser, A. M. J., Marchand, J.-P., Martin, Ph. A.: Droplet dynamics in two-dimensional kinetic Ising model. Helv. Phys. Acta, 55, 259-277 (1982)

[Kes] Kesten, H.: Percolation Theory for Mathematicicians. Boston-Basel: Birkhauser 1982

[KN] Kipnis, C., Newman, C. M.: The metastable behavior of infrequently observed, weakly random, one dimensional diffusion processes. SIAM J. Appl. Math. 45, 972-982 (1985)

[Lig] Liggett, T. M.: Interacting particle systems. Berlin, Heidelberg, New York: Springer 1985

[LS] Lebowitz, J. L. Schonmann, R. H.: On the asymptotics of occurrence times of rare events for stochastic spin systems. J. Stat. Phys. 48, 727-751 (1987)

[NCK] Newman, C. M., Cohen, J. E., Kipnis, C.: Neo-darwinian evolution implies punctuated equilibria. Nature 315, 400-401 (1985)

[MOS] Martinelli, F., Olivieri, E., Scoppola, E.: Small random perturbation of finite and infinite dimensional systems: Unpredicted of exist times. Preprint (1988)

[PL] Penrose, O. Lebowitz, J. L.: Molecular theory of metastability: An update. Appendix to the reprinted edition of the article "Towards a rigorous molecular theory of metastability" by the same authors. In: Fluctuation Phenomena (second edition). Montroll, E. W., Lebowitz, J. L. (eds.) Amsterdam: North-Holland Physics Publishing 1987

[Sch] Schonmann, R. H.: Metastability for the contact process. J. Stat. Phys. 41, 445-464 (1985) 